\title{
Preface of the special issue on global multiobjective optimization
}

\author{
Kaisa Miettinen ${ }^{1}$ (D) Serpil Sayin ${ }^{2}$ (D)
}

Accepted: 20 April 2021 / Published online: 3 May 2021

(c) The Author(s), under exclusive licence to Springer Science+Business Media, LLC, part of Springer Nature 2021

Many real optimization problems have several conflicting objective functions that should be optimized simultaneously. In these multiobjective optimization problems, there are different trade-offs among the objectives and the concept of optimality differs from single objective optimization. This means that there are typically many so-called Pareto optimal solutions. The problems can have different challenges like nonconvex or nondifferentiable functions and different types of variables which lead to the need of global methods.

The call for papers of this special issue was originally announced in relation to the 25th International Conference on Multiple Criteria Decision Making (MCDM 2019) held in Istanbul, Turkey, in June 2019. The International Society on Multiple Criteria Decision Making (www.mcdmsociety.org) organizes these conferences every two years. In addition, manuscripts related to global multiobjective optimization were invited independent from the conference. We received 19 submissions and three of them were accepted for publication after a careful review process.

In the article "Inverse Multiobjective Optimization: Inferring Decision Criteria from Data", Bennet Gebken and Sebastian Peitz work on the reverse question of identifying objectives on which a certain decision is based. They present a way to construct a vector of objective functions of a multiobjective optimization problem so that its extended Pareto optimal set contains a given data set. In this, they consider KKT conditions and derive a system of equations. As application examples, they mention identification of objectives from data and construction of surrogate models for expensive multiobjective optimization problems.

In "Nonconvex Constrained Optimization by a Filtering Branch and Bound", Gabriele Eichfelder, Kathrin Klamroth and Julia Niebling focus on the difficult question of finding feasible solutions to an optimization problem with nonconvex constraints. They introduce a filtering approach and reformulate the constrained nonconvex optimization problem with a single objective as a multiobjective one. The reformulation enables identifying the trade-off

$\bowtie \quad$ Kaisa Miettinen

kaisa.miettinen@jyu.fi

Serpil Sayin

SSAYIN@ku.edu.tr

1 University of Jyvaskyla, Faculty of Information Technology, P.O. Box 35, FI-40014 (Agora), Finland

2 College of Administrative Sciences and Economics, Koç University, 34450 Sariyer, Istanbul, Turkey 
between constraint satisfaction and objective value. They demonstrate the applicability of the approach with numerical tests.

Gabriele Eichfelder and Leo Warnow consider termination criteria to measure the proximity of a solution to the Pareto optimal set in "Proximity Measures based on KKT Points for Constrained Multi-objective Optimization Problems". They propose two proximity measures using the violation of the KKT conditions which is easy to compute. They demonstrate their ideas with three test instances and suggest that their approach can be embedded into algorithms, including evolutionary ones, to improve solution quality.

We would like to express our warmest thanks to all reviewers for their efforts and all researchers who showed interest in this special issue. We would also like to thank the Journal of Global Optimization and the editor-in-chief, Prof. Sergiy Butenko.

Publisher's Note Springer Nature remains neutral with regard to jurisdictional claims in published maps and institutional affiliations. 